\title{
ON THE BLOCH-NEVANLINNA PROBLEM
}

\author{
LIANG-SHIN HAHN
}

\begin{abstract}
In 1929, A. Bloch and R. Nevanlinna [5] posed the problem of whether the derivative of a function of bounded characteristic is also of bounded characteristic. Since then counterexamples have been constructed by Frostman, Rudin, Lohwater, Piranian, Hayman, and Duren ([1], [2], [3], [4], [6], [7]). In this short note we prove that in the Banach space of absolutely convergent power series the subset consisting of members whose derivatives have no finite radial limit in any direction is dense. Since the radial limit of a function of bounded characteristic exists in almost every direction, the result disproves the conjecture in a rather emphatic form. Similar results are established for the space of functions which are analytic in the unit disc and continuous in the closed disc, and also for the space of absolutely convergent Fourier series.

The existential proof given here seems to be easier than any of the known constructive proofs, and actually establishes more.
\end{abstract}

Let $A$ be the Banach space of all power series $f(z)=\sum_{k=0}^{\infty} a_{k} z^{k}$ with $\sum_{k=0}^{\infty}\left|a_{k}\right|<\infty$, endowed with the norm $\|f\|=\sum_{k=0}^{\infty}\left|a_{k}\right|$. Let $E$ be the set consisting of all the functions in $A$ whose derivative has finite radial limit in at least one direction.

LEMMA. E is a set of first category in $A$.

Proof. Let $E_{n}$ be the set of all functions $f$ in $A$ such that for some $\theta$ in $[0,2 \pi]$,

$$
\left|f^{\prime}\left(r e^{i \theta}\right)\right| \leqq n \text { for all } r \in[1-1 / n, 1)
$$

Clearly, $E \subseteq \bigcup_{n=2}^{\infty} E_{n}$. We shall show that, for each $n, E_{n}$ is a nowhere dense set in $A$. This is done in two steps:

$1^{\circ}$. $E_{n}$ is closed. Let $f$ be a function in the closure of $E_{n}$. Then there exists a sequence $\left\{f_{k}\right\}_{k=1}^{\infty}$ of functions $E_{n}$ converging to $f:\left\|f_{k}-f\right\| \rightarrow 0$ $(k \rightarrow \infty)$. Since $f_{k} \in E_{n}$, there corresponds $\theta_{k}$ in $[0,2 \pi]$ such that $\left|f_{k}^{\prime}\left(r \exp \left[i \theta_{k}\right]\right)\right| \leqq n$, for $1-1 / n \leqq r<1$. Since $[0,2 \pi]$ is compact, $\left\{\theta_{k}\right\}_{k=1}^{\infty}$

Received by the editors January 18, 1971 and, in revised form, May 21, 1971.

AMS 1970 subject classifications. Primary 30A12, 30A78.

Key words and phrases. Analytic functions, absolutely convergent power series, radial limits, functions of bounded characteristic, absolutely convergent Fourier series, Abel-Poisson summability, almost uniform convergence.

(C) American Mathematical Society 1972 
has a convergent subsequence. For the sake of simplicity in notation, we may assume that the original sequence $\left\{\theta_{k}\right\}_{k=1}^{\infty}$ itself converges (to $\theta_{0}$, say). Now

$$
\begin{aligned}
\left|f^{\prime}\left(r \exp \left[i \theta_{0}\right]\right)\right| \leqq & \left|f^{\prime}\left(r \exp \left[i \theta_{0}\right]\right)-f^{\prime}\left(r \exp \left[i \theta_{k}\right]\right)\right| \\
& +\left|f^{\prime}\left(r \exp \left[i \theta_{k}\right]\right)-f_{k}^{\prime}\left(r \exp \left[i \theta_{k}\right]\right)\right|+\left|f_{k}^{\prime}\left(r \exp \left[i \theta_{k}\right]\right)\right| .
\end{aligned}
$$

Fix $r, 1-1 / n \leqq r<1$. Given any $\varepsilon>0$, choose $K_{1}$ and $K_{2}$ so large that

and

$$
\left|f^{\prime}\left(r \exp \left[i \theta_{0}\right]\right)-f^{\prime}\left(r \exp \left[i \theta_{k}\right]\right)\right|<\varepsilon / 2, \quad k \geqq K_{1},
$$

$$
\left|f^{\prime}\left(r \exp \left[i \theta_{k}\right]\right)-f_{k}^{\prime}\left(r \exp \left[i \theta_{k}\right]\right)\right|<\varepsilon / 2, \quad k \geqq K_{2} .
$$

The first relation is possible because of the continuity of $f^{\prime}(z)$ in the open unit disc; and the second follows from the uniform convergence of $\left\{f_{k}^{\prime}\right\}_{k=1}^{\infty}$ to $f^{\prime}$ on the circle $|z|=r$. (Note that our norm is stronger than the uniform norm.) Thus

$$
\left|f^{\prime}\left(r \exp \left[i \theta_{0}\right]\right)\right|<\varepsilon / 2+\varepsilon / 2+\left|f_{k}^{\prime}\left(r \exp \left[i \theta_{k}\right]\right)\right| \leqq \varepsilon+n
$$

for $k \geqq \max \left\{K_{1}, K_{2}\right\}$. Now the left-hand side is independent of $k$ and $\varepsilon$ and so we obtain $\left|f^{\prime}\left(r \exp \left[i \theta_{0}\right]\right)\right| \leqq n, 1-1 / n \leqq r<1$, showing that $f \in E_{n}$.

$2^{\circ}$. The complement of $E_{n}$ is everywhere dense. Suppose $f \in A$. We shall show that for any $\varepsilon>0$, there exists $g$ in $A$ but not in $E_{n}$ such that $\|f-g\|<\varepsilon$. Since $E_{n}$ is closed, this will show that $E_{n}$ is nowhere dense. Since

$$
f(z)=\sum_{k=0}^{\infty} a_{k} z^{k}, \quad \sum_{k=0}^{\infty}\left|a_{k}\right|<\infty,
$$

we may choose $N$ so large that $\sum_{k>N}\left|a_{k}\right|<\varepsilon / 2$. Then $\left\|f-g_{1}\right\|<\varepsilon / 2$, where $g_{1}(z)=\sum_{k=0}^{N} a_{k} z^{k}$ is a polynomial. We also have $M=\max _{|z| \leqq 1}\left|g_{1}^{\prime}(z)\right|<\infty$. Next, take an integer $m$ so large that $\varepsilon m / 2>n+M$. Then for this $m$ there exists $\delta>0$ such that

$$
(\varepsilon m / 2) r^{m-1}>n+M \text { if } 1-\delta \leqq r<1 .
$$

Now let $g_{2}(z)=(\varepsilon / 2) z^{m}$. Then $\left\|g_{2}\right\|=\varepsilon / 2$ and $\left|g_{2}^{\prime}\left(r e^{i \theta}\right)\right|=(\varepsilon m / 2) r^{m-1}>n+M$ for all $\theta \in[0,2 \pi]$ if $1-\delta \leqq r<1$. We claim that $g=g_{1}+g_{2}$ satisfies the desired conditions. For, clearly, $g \in A$ and $\|f-g\| \leqq\left\|f-g_{1}\right\|+\left\|g_{2}\right\|<\varepsilon$; moreover,

$$
\left|g^{\prime}\left(r e^{i \theta}\right)\right| \geqq\left|g_{2}^{\prime}\left(r e^{i \theta}\right)\right|-\left|g_{1}^{\prime}\left(r e^{i \theta}\right)\right|>(n+M)-M=n
$$

for all $0 \leqq \theta \leqq 2 \pi$ if $1-\delta \leqq r<1$. Thus $g \notin E_{n}$. Q.E.D.

Being a complete metric space, $A$ is of second category; thus, by the 
Baire category theorem, we obtain

THEOREM 1. The set of all functions $f$ in $A$ such that

is dense in $A$.

$$
\underset{r \rightarrow 1-}{\limsup }\left|f^{\prime}\left(r e^{i \theta}\right)\right|=\infty \quad \text { for all } \theta \in[0,2 \pi]
$$

Let $H C(D)$ be the Banach space of all functions analytic in the unit disc $D$ and continuous in the closed disc $\bar{D}$, equipped with the uniform norm: $\|f\|=\max _{z \in D}|f(z)|, f \in H C(D)$. Then the embedding mapping of $A$ into $H C(D)$ is continuous and $A$ is dense in $H C(D)$; thus we get

THEOREM 2. The set of all functions $f$ in $H C(D)$ such that

$$
\limsup _{r \rightarrow 1-}\left|f^{\prime}\left(r e^{i \theta}\right)\right|=\infty \quad \text { for all } \theta \in[0,2 \pi]
$$

is dense in $H C(D)$.

Let $A(T)$ be the Banach space of absolutely convergent Fourier series $f(\theta)=\sum_{k=-\infty}^{\infty} a_{k} e^{i k \theta}$ with the norm $\|f\|=\sum_{k=-\infty}^{\infty}\left|a_{k}\right|$.

THEOREM 3. The set of all functions $f$ in $A(T)$ such that $\sum_{k=-\infty}^{\infty} k a_{k} e^{i k \theta}$ is Abel-Poisson summable for no value of $\theta$ is dense in $A(T)$.

Proof. The proof is almost the same as before. Let $E_{n}$ be the set of all functions $f(\theta)=\sum_{k=-\infty}^{\infty} a_{k} e^{i k \theta}$ in $A$ such that there exists $\theta$ in $[0,2 \pi]$ with

$$
\left|\sum_{k=-\infty}^{\infty} k a_{k} r^{|k|} e^{i k \theta}\right| \leqq n \text { for } r \in[1-1 / n, 1) .
$$

It suffices to show that each $E_{n}$ is nowhere dense. To show that $E_{n}$ is closed, observe that if $f(\theta)=\sum_{k=-\infty}^{\infty} a_{k} e^{i k \theta}$ is a member of $A(T)$, the function $\sum_{k=-\infty}^{\infty} k a_{k} r^{|k|} e^{i k \theta}$ is continuous for $z=r e^{i \theta}$ in the unit disc, and the convergence of $f_{j}(\theta)=\sum_{k=-\infty}^{\infty} a_{k}^{(j)} e^{i k \theta}$ to $f(\theta)=\sum_{k=-\infty}^{\infty} a_{k} e^{i k \theta}$ in the norm of $A(T)$ (as $j \rightarrow \infty)$ implies the almost uniform convergence of $\sum_{k \geqq 0} k a_{k}^{(j)} z^{k}$ and $\sum_{k>0} k a_{-k}^{(j)} \bar{z}^{k}$ to $\sum_{k \geqq 0} k a_{k} z^{k}$ and $\sum_{k>0} k a_{-k} \bar{z}^{k}$, respectively, in the unit disc. Therefore, if $f$ is a member of the closure of $E_{n}$, by using the triangle inequality one can easily show that $f$ actually belongs to $E_{n}$; i.e., $E_{n}$ is closed. The rest of the proof is a repetition of the previous argument.

In conclusion we note that the "multiplier" $\{k\}$ may be replaced by $\{|k|\}$ in Theorem 3.

\section{REFERENCES}

1. P. Duren, On the Bloch-Nevanlinna conjecture, Colloq. Math. 20 (1969), 295-297. MR 39 \#5805.

2. O. Frostman, Sur les produits de Blaschke, Proc. Roy. Physiog. Soc. Lund. 12 (1942), 169-182. MR 6, 262. 
3. W. Hayman, On the characteristic of functions meromorphic in the unit disc and of their integrals, Acta Math. 112 (1964), 181-214. MR 29 \#6019.

4. A. J. Lohwater, G. Piranian and W. Rudin, The derivative of a schlicht function, Math. Scand. 3 (1955), 103-106. MR 17, 249.

5. R. Nevanlinna, Le théorème de Picard-Borel et la théorie des fonctions meromorphes, Gauthiers-Villars, Paris, 1929, p. 138.

6. W. Rudin, On a problem of Bloch and Nevanlinna, Proc. Amer. Math. Soc. 6 (1955), 202-204. MR 16, 810.

7. - On the radial variation of analytic functions, Duke Math. J. 22 (1955), 235-242. MR 18, 27.

Department of Mathematics and Statistics, University of New Mexico, Albuquerque, New Mexico 87106 